\title{
BMJ Open Atrial Fibrillation Screen, Management And Guideline Recommended Therapy (AF SMART II) in the rural primary care setting: an implementation study protocol
}

\author{
Jessica J Orchard, ${ }^{1}$ Lis Neubeck, ${ }^{2}$ Ben Freedman, ${ }^{1,3}$ Ruth Webster, ${ }^{4}$ \\ Anushka Patel, ${ }^{4}$ Robyn Gallagher, ${ }^{5}$ Jialin Li, ${ }^{5}$ Charlotte Mary Hespe, ${ }^{6}$ \\ Caleb Ferguson, ${ }^{7}$ Nicholas Zwar, ${ }^{8}$ Nicole Lowres ${ }^{1,3}$
}

To cite: Orchard JJ, Neubeck L, Freedman B, et al. Atrial Fibrillation Screen, Management And Guideline Recommended Therapy (AF SMART II) in the rural primary care setting: an implementation study protocol. BMJ Open 2018;8:e23130. doi:10.1136/ bmjopen-2018-023130

- Prepublication history for this paper is available online. To view these files, please visit the journal online (http://dx.doi. org/10.1136/bmjopen-2018023130).

Received 22 March 2018

Revised 25 July 2018

Accepted 26 September 2018

Check for updates

(C) Author(s) (or their employer(s)) 2018. Re-use permitted under CC BY-NC. No commercial re-use. See rights and permissions. Published by BMJ.

For numbered affiliations see end of article.

Correspondence to

Jessica J Orchard;

jessica.orchard@sydney.edu.au

\section{ABSTRACT}

Introduction Screening for atrial fibrillation (AF) in people $\geq 65$ years is now recommended by guidelines and expert consensus. While AF is often asymptomatic, it is the most common heart arrhythmia and is associated with increased risk of stroke. Early identification and treatment with oral anticoagulants can substantially reduce stroke risk. The general practice setting is ideal for opportunistic screening and provides a natural pathway for treatment for those identified. This study aims to investigate the feasibility of implementing screening for AF in rural general practice using novel electronic tools. It will assess whether screening will fit within an existing workflow to quickly and accurately identify AF, and will potentially inform a generalisable, scalable approach.

Methods and analysis Screening with a smartphone ECG will be conducted by general practitioners and practice nurses in rural general practices in New South Wales, Australia for 3-4 months during 2018-2019. Up to 10 practices will be recruited, and we aim to screen 2000 patients aged $\geq 65$ years. Practices will be given an electronic screening prompt and electronic decision support to guide evidence-based treatment for those with AF. De-identified data will be collected using a clinical audit tool and qualitative interviews will be conducted with selected practice staff. A process evaluation and cost-effectiveness analysis will also be undertaken. Outcomes include implementation success (proportion of eligible patients screened, fidelity to protocol), proportion of people screened identified with new AF and rates of treatment with anticoagulants and antiplatelets at baseline and completion. Results will be compared against an earlier metropolitan study and a 'control' dataset of practices.

Ethics and dissemination Ethics approval was received from the University of Sydney Human Research Ethics Committee on 27 February 2018 (Project no.: 2017/1017). Results will be disseminated through various forums, including peer-reviewed publication and conference presentations.

Trial registration number ACTRN12618000004268; Preresults.
Strengths and limitations of this study

- New technology is used to quickly and efficiently identify and treat atrial fibrillation (AF), within an existing workflow in the general practice setting.

- General practices are ideally placed to screen for and diagnose AF, as well to provide a pathway to care for those identified during screening.

- Conducting the study in the rural setting will inform future AF screening policies to extend the evidence base beyond metropolitan settings.

- A potential limitation is the small size and non-randomised design, which would ideally be overcome in a future large-scale study.

\section{INTRODUCTION}

Atrial fibrillation (AF) is the most common heart arrhythmia, affecting 33.5 million people worldwide. ${ }^{1}$ A person's likelihood of developing $\mathrm{AF}$ increases with age, reaching $37 \%$ for those aged $\geq 55$ years. ${ }^{2}$ Importantly, $\mathrm{AF}$ is associated with a fivefold increase in risk of stroke. ${ }^{34}$ However, $1.4 \%-1.6 \%$ of the population aged $\geq 65$ have undiagnosed $\mathrm{AF}^{56}{ }^{5}$ which is commonly asymptomatic. ${ }^{78}$ Stroke caused by AF is largely preventable with appropriate oral anticoagulant (OAC) therapy, which can reduce AF-related stroke risk by $64 \% .^{9}$ Although OAC is strongly recommended by clinical practice guidelines for those with additional stroke risk factors, as set out in the $\mathrm{CHA}_{2} \mathrm{DS}_{2}$-VASc score, ${ }^{10}{ }^{11}$ there has historically been a large gap between evidence and practice with only $60 \%$ of eligible patients prescribed OAC. ${ }^{12}$ Recently, there are signs of improvement, with a recent Public Health England report noting $77.9 \%$ of eligible patients with AF in England were treated with OAC. ${ }^{13}$ Similar increases in OAC prescription, 
largely due to the introduction of novel OACs, have been reported in Spain and Denmark. ${ }^{14} 15$

Screening for AF in people $\geq 65$ years by opportunistic pulse palpation or ECG rhythm strip is now recommended by clinical practice guidelines and international expert consensus. ${ }^{11} 1617$ Many studies have demonstrated the effectiveness of opportunistic screening for AF in general practice. The SAFE study demonstrated that opportunistic screening of patients aged $\geq 65$ years by general practitioners (GPs), with a flag prompt on the patient file, was an effective screening method. ${ }^{5} \mathrm{AF}$ screening in general practice was found to be promising during influenza vaccination in the Netherlands, ${ }^{18}$ and was successfully performed by practice nurses in the UK. ${ }^{19}$ Our previous research showed AF screening by practice nurses to be feasible, both opportunistically and during the influenza vaccination period. ${ }^{20} 21$

With $84 \%$ of Australian general practices employing practice nurses, ${ }^{22}$ there is scope for AF screening to be performed during existing practice nurse health appointments. Ideally, practice nurses could screen patients for AF during the influenza or shingles vaccination and/or coordinated chronic care consultations. These appointments are already funded by the Australian Government: the influenza vaccination is recommended annually and provided free for patients aged $\geq 65$ years $^{23}$; shingles vaccines will be provided free for people aged $\geq 70$ with a 5-year catch-up programme for people aged 71-7924; annual health assessments are funded for those aged $\geq 75^{25}$; and annual coordination consultations are funded by Medicare for patients with chronic conditions (eg, diabetes, cardiovascular) on a care plan. ${ }^{26}$ These are all occasions when patients in the target age group are likely to be available and receptive to AF screening, in an appropriate setting.

While numerous studies have been conducted in metropolitan areas, there is a paucity of data regarding AF screening in rural areas. A recent systematic review of AF screening studies conducted in rural settings acknowledged the need for more information on AF burden and risk factors specifically in rural areas. ${ }^{27}$ In the Australian context, it is known that people living in rural areas have worse cardiovascular outcomes. ${ }^{28}$ It has been reported that there is a lack of evidence about rural cardiovascular disease prevention and 'characteristics associated with quality of care', which highlights the need for more programmes in rural primary care, especially for highrisk patients. ${ }^{29}$ This study aims to implement a screening programme with a quality improvement (QI) focus, to better inform future AF screening policies to extend the evidence base beyond metropolitan settings.

\section{METHODS AND ANALYSIS \\ Design}

This study, Atrial Fibrillation Screen, Management And Guideline Recommended Therapy (AF-SMART II), will investigate the feasibility of implementation of an $\mathrm{AF}$ screening and use of an AF electronic decision support tool (EDS), in rural general practices. It is a cross-sectional, observational implementation study of $\mathrm{AF}$ screening using a smartphone ECG conducted by GPs and practice nurses in up to 10 rural general practices in New South Wales (NSW), Australia. It is very similar to the study protocol used in the AF-SMART study which was conducted in eight metropolitan general practices in Sydney, Australia from 2016 to 2018. AF-SMART II is a rural extension of AF-SMART with some important modifications based on feedback obtained in the metropolitan study. These modifications include: (1) an improved electronic screening prompt to improve the proportion of eligible patients screened; (2) the introduction of GP continuing professional development (CPD)/QI points to provide additional incentives for GPs; (3) a more structured approach to nurse screening and (4) a shorter screening period.

\section{Practice recruitment}

A convenience sample of up to 10 rural general practices, recruited through several NSW Primary Health Networks, will be invited to participate in the study. Practices will be required to:

- Have at least one practice nurse.

- Use either 'Best Practice' or 'Medical Director' as the practice electronic patient record management system.

- Be willing to have 'TopBar' (PenCS third party software) and the clinical audit tool 'PenCAT' for data collection installed (licences are provided by the Primary Health Networks, NSW).

\section{- Have WiFi.}

\section{Preintervention: training and set up electronic study tools}

Once a practice has been recruited, the preintervention phase will involve setting up the electronic study tools and relevant training. Each practice will be provided with several Kardia smartphone ECG (iECG) devices to screen for AF (figure 1). This device has approval from the Australian Therapeutic Goods Administration as a Medical Device, Class IIa and has a validated, automated algorithm for detecting AF with 95\% sensitivity and 99\% specificity. $^{21}$

In addition, practices will be provided with two custom-designed apps that were developed and tested in the AF-SMART study (figures 2 and 3). These apps are the:

1. AF app with screening prompt, which extracts data from the electronic patient record and provides a prompt for AF screening when an eligible patient's file is opened, and allows clinical staff to record the provisional screening results (figures 2 and 3 ).

2. EDS system, which guides evidence-based treatment of those diagnosed with AF. The EDS is a clinically validated QI tool, designed to bridge gaps between evidence and practice in AF risk management. ${ }^{30}$ The EDS extracts data from electronic patient records 


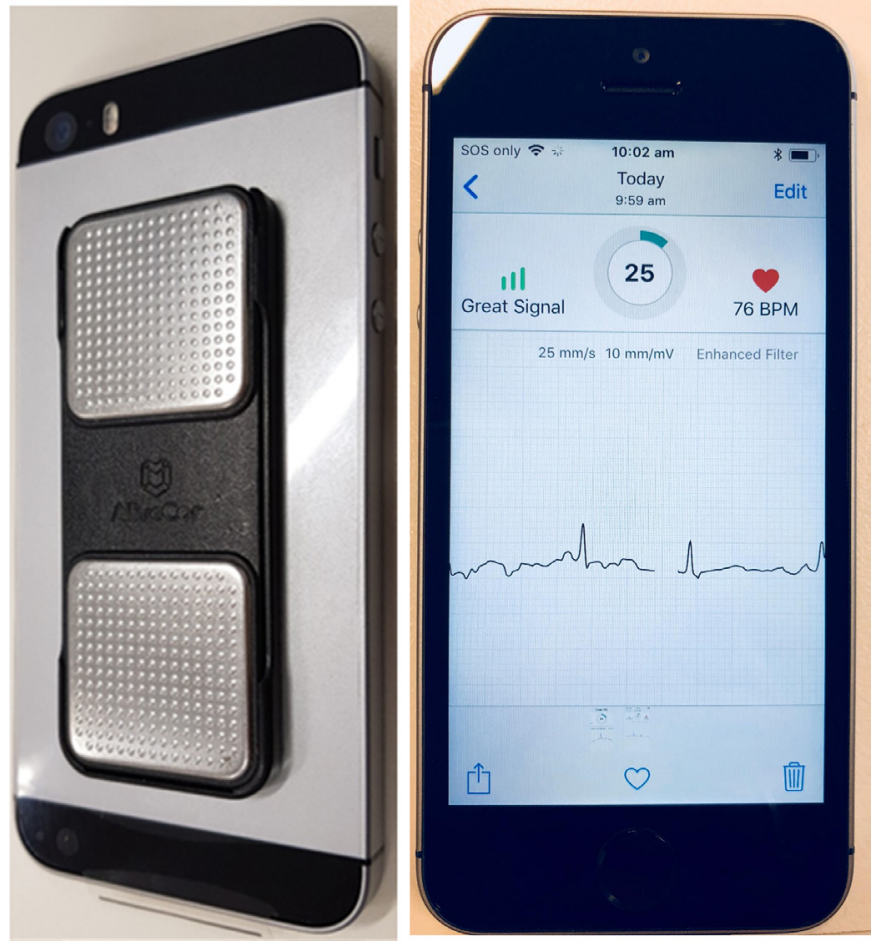

Figure 1 Kardia device and iECG reading.

regarding current $\mathrm{OAC}$ and antiplatelet prescriptions and calculates individual stroke risk scores $\left(\mathrm{CHA}_{2} \mathrm{DS}_{2}-\right.$ VASc) (figure 4). It then provides clinical staff with evidence-based medication guidelines to support decisions on OAC prescription.

The apps are located in the TopBar hosting platform that integrates with the general practice electronic patient record management systems.

Once the technology set-up is complete, practice nurses and GPs will receive training on the use of the electronic tools and iECG as well as recent developments in evidence-based management of AF for stroke prevention, highlighting guideline recommendations for $\mathrm{OAC}$, and recommendations against using aspirin to treat AF. ${ }^{11}$ Practices will be provided with posters for the reception area advertising the study and a laminated participant information statement will be available at reception.

\section{Patient eligibility}

All patients aged $\geq 65$ years presenting to the general practice for annual influenza vaccination, shingles vaccination, chronic care assessment or seeing their GP, will be eligible for $\mathrm{AF}$ screening if they meet the following criteria:

- Age $\geq 65$ years.

- No current recorded diagnosis of AF.

- Have not already been screened with the iECG in the past 12 months.

Patients with terminal illness and patients unable to provide informed consent will be excluded.

\section{Screening protocol}

The screening intervention will be conducted for a period of 3-4 months in each practice. The process is shown in figure 5, and summarised below:

- The AF screening prompt will notify clinical staff a patient is eligible for iECG screening.

- The GP/nurse will explain the screening process and purpose, and obtain informed oral consent from patients.

- The GP/nurse will record a 30 s iECG. The iECG trace will be visible in real time on the phone, and a pdf will also be available to download from the secure Kardia website. An automated interpretation ('Possible AF', 'Normal' or 'Unclassified') is provided immediately after successful recording.

- The GP/nurse will enter the iECG screening result into the AF app in TopBar.

- Depending on the iECG result, follow the appropriate protocol.

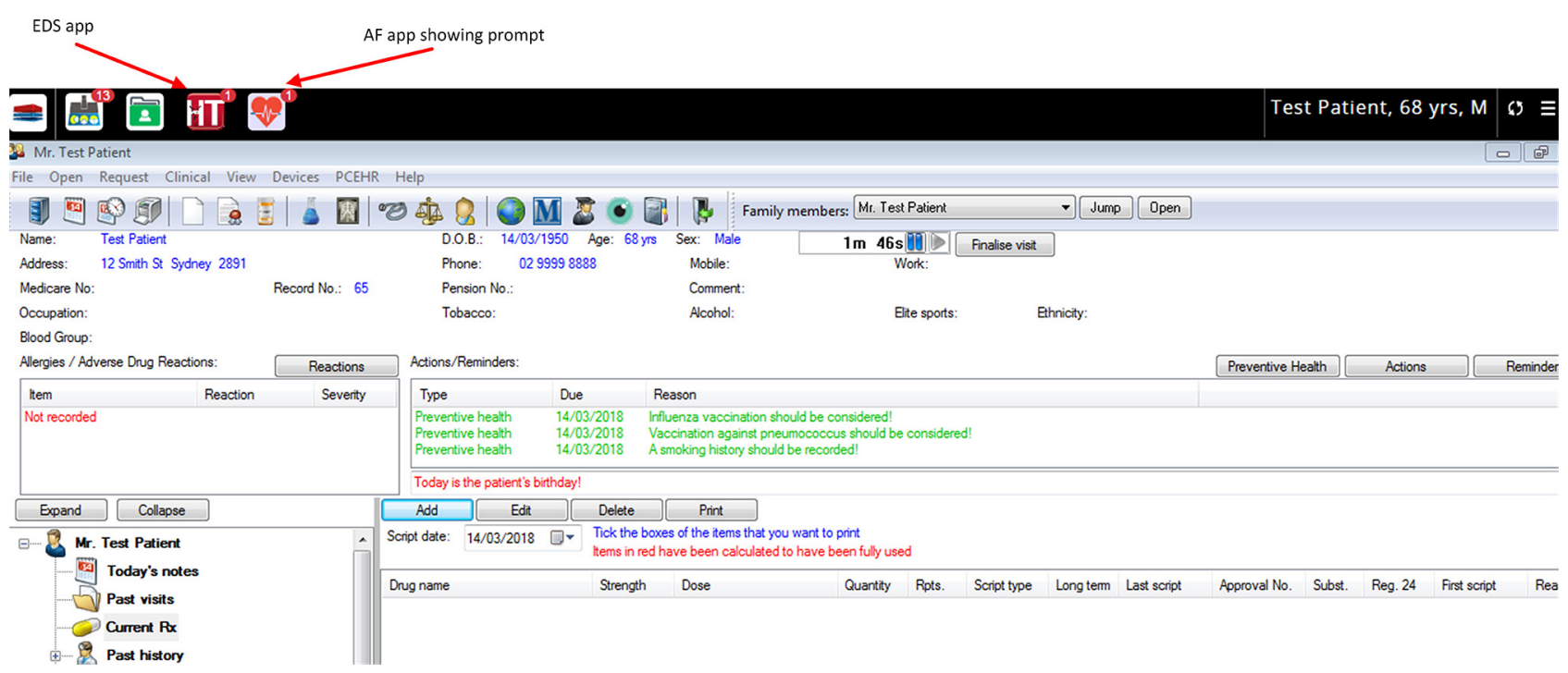

Figure 2 Screenshot showing test patient file and TopBar apps. AF, atrial fibrillation. 


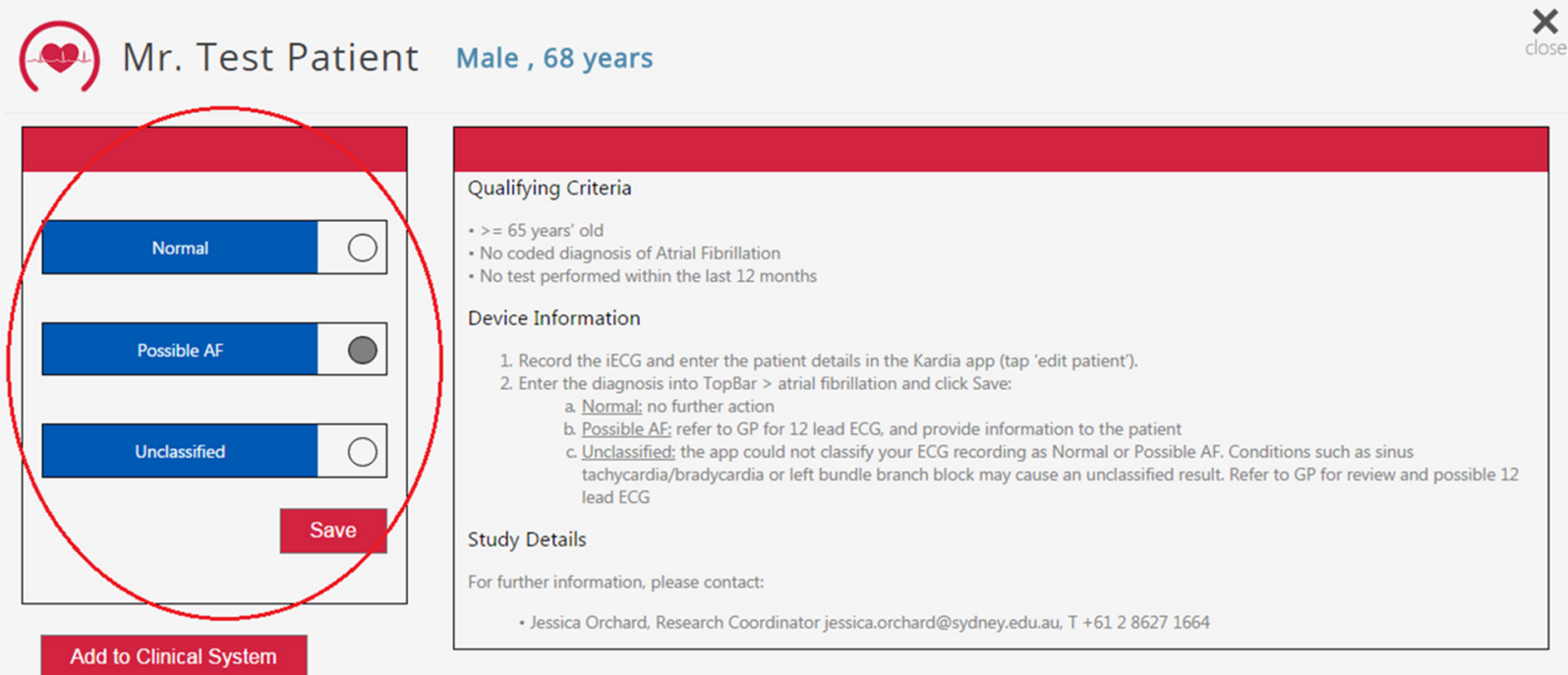

Figure 3 entering an iECG result in TopBar AF app (test patient shown). AF, atrial fibrillation; GP, general practitioner.

\section{Protocol if a patient receives 'possible AF' diagnosis}

The GP/nurse will inform the patient of the diagnosis and give a brief explanation. If the nurse performed the screening, the patient will be referred to their GP. Further investigation and management is at the GP's discretion, which could include referral to a specialist. A 12-lead ECG is recommended to provide additional confirmation for all new $\mathrm{AF}$ diagnoses, and to add extra leads for $\mathrm{AF}$ diagnostic workup. In some cases of paroxysmal AF, the 12-lead ECG may show sinus rhythm while the iECG showed AF. This will be obvious from a comparison of the p-waves on the lead I iECG and lead I of the 12-lead ECG, as well as the regularity of the rhythm. Following a 12-lead ECG, for patients with confirmed AF, GPs will be encouraged to use the EDS to

\section{国范国國所 \\ Atrial Fibrillation \\ $\mathrm{CHA}_{2} \mathrm{DS}_{2}$-VASc score: 5 \\ 5-year stroke risk: > 33\% \\ Stroke risk is high due to AF. Oral anticoagulant therapy is indicated if there are no absolute contraindications. \\ This patient is eligible for PBS subsidised NOAC (Apixaban, Dabigatran, Rivaroxaban) if patient does not have valvular AF (rheumatic mitral valve disease or mechanical valve replacement) \\ Antiplatelet therapy may be indicated if oral anticoagulant treatment is not commenced. NB antiplatelet therapy does not signifcantly reduce the risk of stroke in $\mathrm{AF}$}

\section{Stroke Risk Calculation}

AF significantly increases risk of stroke - these are more severe and more often fatal than strokes from other causes. Most guidelines now recommend use of the $\mathrm{CHA}_{2} \mathrm{DS}_{2}$-VASc score to estimate stroke risk. The score differs from the older $\mathrm{CHADS}_{2}$ as it includes more risk factors.

The $\mathrm{CHA}_{2} \mathrm{DS}_{2}$-VASc score includes the following risk factors:

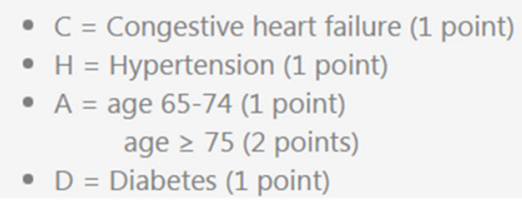

Figure 4 Screenshot showing EDS recommendation for test patient. AF, atrial fibrillation; NOAC, novel oral anticoagulant. 


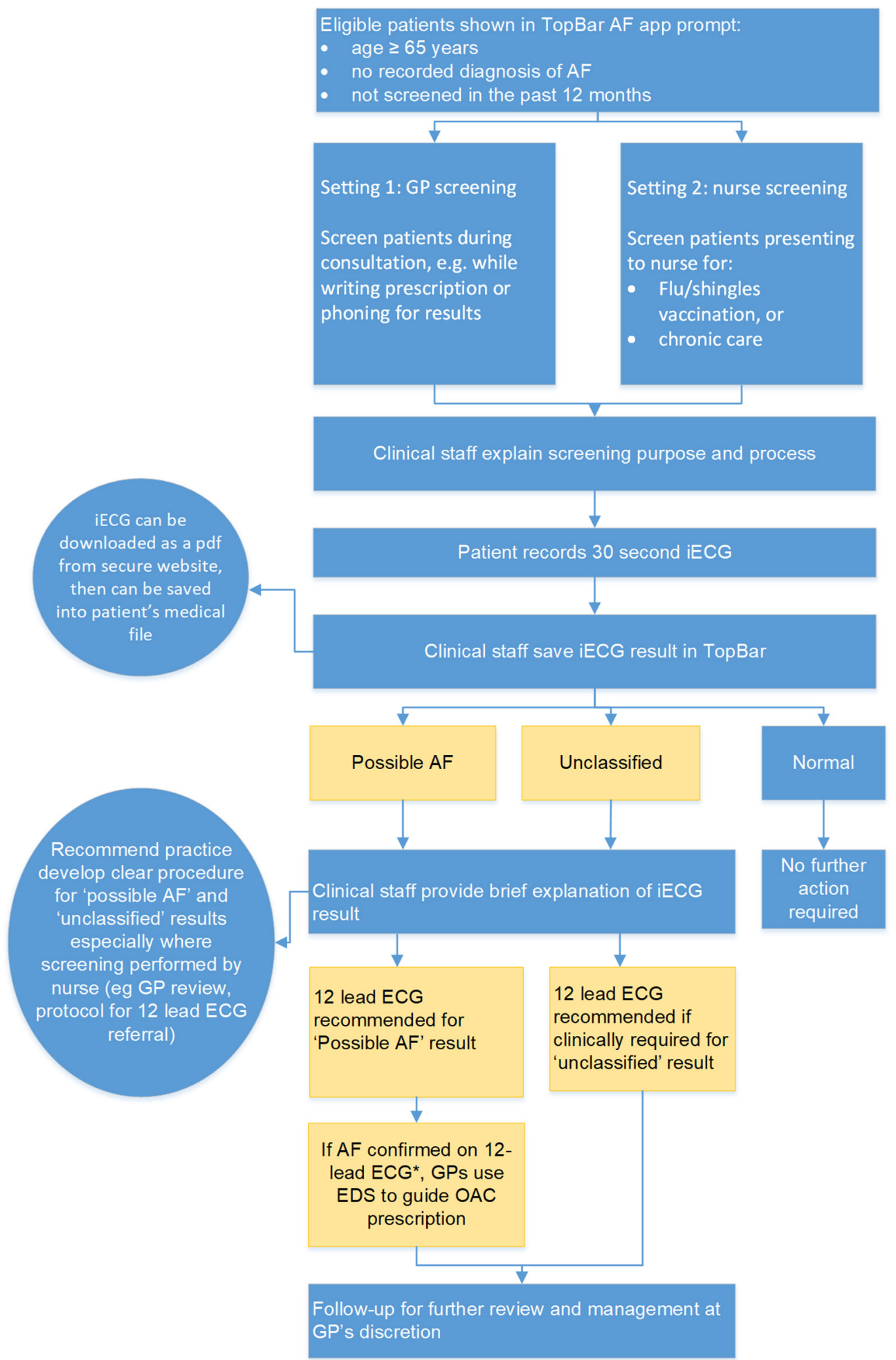

* Occasionally, there is a delay between the rhythm strip and 12-lead ECG during which the rhythm has changed from AF to sinus rhythm. In this scenario, a comparison of the absence of $p$ waves in the screening Lead I rhythm strip and the $p$ waves in the Lead I of the 12-lead ECG can be very useful to confirm the rhythm during screening was actually AF.

Figure 5 Process flow of AF screening. AF, atrial fibrillation; GP, general practitioner; OAC, oral anticoagulant.

review each patient's $\mathrm{CHA}_{2} \mathrm{DS}_{2}$-VASc score and commence guideline-recommended treatment to reduce stroke risk.

\section{Protocol if a patient receives 'normal' diagnosis}

The GP/nurse will inform the patient of the diagnosis. No further action is required.

\section{Protocol if a patient receives 'unclassified' diagnosis}

The GP/nurse will inform the patient of the diagnosis and give a brief explanation based on information provided by researchers. Depending on individual patient's history and iECG, a 12-lead ECG may be recommended by the GP but follow-up is at the GP's discretion. There are a 
number of conditions that can lead to this particular diagnosis (eg, sinus tachycardia/bradycardia, left bundle branch block, right bundle branch block or multiple ectopic beats) which may or may not be clinically significant, or previously known.

\section{CPD and QI points}

In order to assist in practice recruitment, education and training will be structured to be eligible for CPD points for nurses and GPs. In addition, all practices will be encouraged to use the research project to conduct an $\mathrm{AF}$ QI programme that will attract specific QI professional development points.

\section{Study outcomes}

- Implementation success through process measures including proportion of eligible patients seen in the practice during the period who are screened (calculated from information available in the de-identified data extracts), fidelity to the protocol and time taken to complete the intervention.

- Proportion of people screened identified with new AF.

- Proportion of patients where EDS page accessed.

- Proportion of eligible patients prescribed OAC as a result of the intervention.

- Proportion of patients with diagnosed AF prescribed antiplatelet therapy during the study period.

- Prevalence of AF at baseline (compared with metropolitan and control group, as defined below).

- Incidence of new AF at completion of the intervention (compared with metropolitan and control group).

- Rates of treatment with anticoagulants and antiplatelets at baseline and at completion of the intervention (compared with metropolitan and control group).

- Acceptability, competing demands, barriers and enablers according to staff involved in the intervention.

- Cost-effectiveness analysis (ie, incremental cost-effectiveness ratio (ICER) of screening per quality-adjusted life year (QALY) gained and per stroke avoided), using data from Cadilhac et $a l^{31}$ to estimate the present value of QALYs gained for each ischaemic stroke prevented.

\section{Data collection and sample size}

The study aims to screen approximately 2000 patients in total from up to 10 general practices. Assuming an incidence of $1.4 \%$ of people aged $\geq 65$ with unknown $\mathrm{AF}^{32}$ we estimate 28 new cases of AF would be identified. A sample size of 2000 , assuming $1.4 \%$ incidence, would provide a $95 \%$ CI of $0.93 \%$ to $2.02 \%$ (18-40 cases).

PenCAT will be configured to collect de-identified data from electronic patient records. These data include demographic, medication and diagnostic information. Data extracts will be taken at baseline, end of month 1 , end of month 2 and end of month 3. The screening period will be extended if required so that practices get all four data extracts. Following each data extract, feedback will be provided to practices including a summary of results (number screened by each staff member in total and for that month).
Following completion of the iECG screening period, selected staff from each practice will be interviewed to gather their feedback as part of a comprehensive process evaluation. Semistructured interviews with selected GPs, practice nurses and practice managers will be carried out by researchers at the end of the screening intervention. The interviews will be audio recorded and transcribed.

\section{Statistical analysis}

\section{Quantitative analysis}

Descriptive analyses will be carried out both at the individual practice level and all practices pooled together. Data collected in this study will be compared with data collected from the earlier study in metropolitan Sydney, and a database of 'control' practices with the same data-parameters collected through concurrent studies run by The George Institute. Analyses of these data will be conducted using SAS v 9.4, with $\chi^{2}$ tests used to test associations between AF incidence and method of detection (screen detected or clinically detected), and two-tail $p$ values $<0.05$ will be considered significant.

\section{Cost-effectiveness analysis}

A basic economic model for AF screening has previously been developed and used in the SEARCH-AF ${ }^{33}$ study. This model will be adapted and extended to evaluate screening for $\mathrm{AF}$ by the practice nurse in the general practice. A modelled cost-effectiveness analysis from an Australian health funder perspective will be performed comparing the cost of iECG population-based screening for AF, to diagnosed $\mathrm{AF}$ in an unscreened population of Australian men and women aged $65-84$ years. Future costs will be discounted at a rate of $5 \%$. The results will be expressed as an ICER per stroke avoided and per QALY gained.

Sensitivity analyses will be performed, including varying the base assumptions of a guideline-adherence rate for OAC prescription from $40 \%$ to $90 \%$ and increasing the cost of treatment. We will obtain 95\% CIs for all ICERs using a multifactorial Monte Carlo simulation ${ }^{34}$ as described in SEARCH-AF. ${ }^{33}$

\section{Qualitative analysis}

A detailed process evaluation using mixed methods will be undertaken to evaluate the iECG screening process. Realist evaluation ${ }^{35}$ will be used to analyse the interaction of context, mechanisms for change and the outcomes that are produced in both this study and the metropolitan component. In this way, we will piece together a detailed understanding of how the intervention influenced the capacity, opportunity and motivation of providers to improve detection and evidence-based treatment of AF.

\section{Patient involvement}

Development and refinement of the protocol was an iterative process. Feedback obtained through interviews patients, nurses, GPs and practice managers about their experience and preferences during previous $\mathrm{AF}$ screening studies ${ }^{2021}$ contributed to the development of the protocol. Following the study, participating practices 
will be provided with a summary of the findings of the study, together with a full copy of any publications, which will be made available to patients in the practice reception area.

\section{ETHICS AND DISSEMINATION}

The study will comply with the National Health and Medical Research Council ethical guidelines. Participating practices will provide written, informed consent and patients being screened will provide oral consent. We used the Standard Protocol Items: Recommendations for Interventional Trials checklist when writing our protocol report. ${ }^{36}$ The study will be administered by the Charles Perkins Centre, University of Sydney. Results of the study will be disseminated through various forums, including peer-reviewed publications and presentation at national and international conferences.

\section{CONCLUSIONS}

Screening for AF in people $\geq 65$ years is now recommended by guidelines and international expert consensus. ${ }^{11} 16$ Importantly, a comprehensive screening programme should include a system to support evidencebased treatment for patients ultimately diagnosed with the condition. ${ }^{16}$ General practices are well placed to opportunistically screen older patients, and are ideal in terms of the pathway for treatment for those identified. This study will provide electronic prompts for screening and test a patient-level EDS integrated with the practice's clinical software, to support evidence-based treatment.

In previous studies, a range of evidence-based interventions has been developed to increase effective prescribing of guideline-recommended OAC in primary care settings. These include use of EDS tools, ${ }^{37}$ targeted GP-education programmes ${ }^{38}$ consultant-led primary care anticoagulation assessment clinics ${ }^{39}$ and patient-focused education interventions. ${ }^{40}$ Overall, these interventions increase quality prescribing, but the results are varied in terms of effect size and duration. One study with a large effect size used a consultant-led anticoagulation assessment within the general practice to identify and review patients with $\mathrm{AF}$ with a $\mathrm{CHA}_{2} \mathrm{DS}_{2}$-VASc score $\geq 1$ who were 'suboptimally anticoagulated'. ${ }^{39}$ This study reported a substantial and significant increase in appropriate OAC treatment from $77 \%$ to $95 \%(\mathrm{p}<0.0001)$, suggesting that an intensive, systematic approach, together with numerous reminders to improve patient attendance at in-person reviews, can be very successful in increasing OAC uptake. ${ }^{39}$ In contrast, a cluster-randomised study providing doctors with patientlevel recommendations via an EDS did not show significant improvements in evidence-based OAC treatment. ${ }^{37}$

This study aims to investigate the feasibility of implementing screening for AF in rural general practice. It follows on from the first phase of the study conducted in metropolitan general practice, allowing comparison against a metropolitan setting and a control group of standard practices. The project will assess if screening will efficiently fit within an existing workflow to quickly and accurately identify AF. The study will also explore the effect of GP and practice nurse education, electronic screening prompts and EDS to facilitate evidence-based treatment.

In Australia, the Heart Foundation's Guideline for the Diagnosis and Management of Atrial Fibrillation will be released in August 2018, the draft guideline recommends opportunistic screening for AF in general practice for people aged $\geq 65$ years. This study will potentially have important implications for the implementation of a large scale opportunistic AF screening programme for those aged $\geq 65$ years. It could also inform the design of a future randomised trial of AF screening. It is anticipated that the study will demonstrate that the general practice setting, provided with additional electronic tools, can meet the challenges of large-scale opportunistic screening with a cheap, convenient, scalable solution.

\section{Author affiliations}

${ }^{1}$ Sydney Medical School and Charles Perkins Centre, University of Sydney, Sydney, New South Wales, Australia

${ }^{2}$ School of Health and Social Care, Edinburgh Napier University, Edinburgh, UK ${ }^{3}$ Heart Research Institute, Sydney, New South Wales, Australia

${ }^{4}$ The George Institute for Global Health, University of New South Wales, Newtown, New South Wales, Australia

${ }^{5}$ Sydney Nursing School, University of Sydney, Sydney, New South Wales, Australia ${ }^{6}$ General Practice Research, School of Medicine, The University of Notre Dame, Sydney, New South Wales, Australia

${ }^{7}$ Western Sydney Nursing and Midwifery Centre, Western Sydney University and Western Sydney Local Health District, Sydney, New South Wales, Australia ${ }^{8}$ School of Medicine, Faculty of Science, Medicine and Health, University of Wollongong, Wollongong, New South Wales, Australia

Contributors LN, BF, NL and JO conceived the original concept of the study. JO, LN, NL, BF, CMH, CF, RG and NZ were involved in the design of the study. LN provided qualitative analysis expertise and NL provided cost-effectiveness analysis expertise. RW and AP were involved in the design and testing of the electronic study apps and provided statistical expertise. JO, LN, BF, JL and NL were involved in drafting the article. $\mathrm{CMH}$ and NZ provided input on general practice aspects of study design, including in relation to educational materials for practices. J0, LN, BF, $\mathrm{RW}, \mathrm{AP}, \mathrm{RG}, \mathrm{JL}, \mathrm{CF}, \mathrm{NZ}$ and NL contributed to the critical revision of the drafting and final approval of the manuscript.

Funding This work was supported by a National Heart Foundation of Australia/ New South Wales Health Cardiovascular Research Network Research Development Project Grant (101133) with top-up funding to complete the study in rural NSW through an investigator-initiated grant from Pfizer. AliveCor has provided free Kardia devices for study purposes. J0 is supported by an Australian Government Research Training Program (RTP) Scholarship. RW is funded by an NHMRC Early Career Fellowship (APP1125044). NL is funded by an NSW Health Early Career Fellowship (H16/ 52168).

Disclaimer The funders have no influence on study design, data collection or the decision to submit reports for publication.

Competing interests NL and BF report grants from BMS/Pfizer during the conduct of the study. RW and AP report other financial interests from George Health, outside the submitted work. CF reports personal fees from Pfizer outside the submitted work. LN reports grants from Pfizer/BMS and Boehringer Ingelheim outside the submitted work. BF reports prior fees and advisory board honoraria from Bayer Pharma AG, Boehringer Ingelheim, and BMS/Pfizer but for the past year has removed himself from pharmaceutical advisory boards and receives speaker fees only for accredited educational meetings.

Patient consent Not required. 
Ethics approval Approval for the study was received from the University of Sydney Human Research Ethics Committee on 27 February 2018 (Project no.: 2017/017).

Provenance and peer review Not commissioned; externally peer reviewed.

Open access This is an open access article distributed in accordance with the Creative Commons Attribution Non Commercial (CC BY-NC 4.0) license, which permits others to distribute, remix, adapt, build upon this work non-commercially, and license their derivative works on different terms, provided the original work is properly cited, appropriate credit is given, any changes made indicated, and the use is non-commercial. See: http://creativecommons.org/licenses/by-nc/4.0/.

\section{REFERENCES}

1. Chugh SS, Havmoeller R, Narayanan K, et al. Worldwide epidemiology of atrial fibrillation: a global burden of disease 2010 study. Circulation 2014;129:837-47.

2. Weng LC, Preis SR, Hulme OL, et al. Genetic predisposition, clinical risk factor burden, and lifetime risk of atrial fibrillation. Circulation 2018;137:1027-38.

3. Hughes M, Lip GY, et al. Guideline Development Group. Stroke and thromboembolism in atrial fibrillation: a systematic review of stroke risk factors, risk stratification schema and cost effectiveness data. Thromb Haemost 2008;99:295-304.

4. Wolf PA, Abbott RD, Kannel WB. Atrial fibrillation as an independent risk factor for stroke: the Framingham Study. Stroke 1991;22:983-8.

5. Fitzmaurice DA, Hobbs FD, Jowett S, et al. Screening versus routine practice in detection of atrial fibrillation in patients aged 65 or over: cluster randomised controlled trial. BMJ 2007;335:383.

6. Lowres N, Neubeck L, Redfern J, et al. Screening to identify unknown atrial fibrillation. A systematic review. Thromb Haemost 2013;110:213-22.

7. Lee J, Reyes BA, McManus DD, et al. Atrial fibrillation detection using an iPhone 4S. IEEE Trans Biomed Eng 2013;60:203-6.

8. Savelieva I, Camm AJ. Clinical relevance of silent atrial fibrillation: prevalence, prognosis, quality of life, and management. J Interv Card Electrophysiol 2000;4:369-82.

9. Hart RG, Pearce LA, Aguilar MI. Meta-analysis: antithrombotic therapy to prevent stroke in patients who have nonvalvular atrial fibrillation. Ann Intern Med 2007;146:857-67.

10. January CT, Wann LS, Alpert JS, et al. 2014 AHA/ACC/HRS guideline for the management of patients with atrial fibrillation: a report of the American College of Cardiology/American Heart Association Task Force on Practice Guidelines and the Heart Rhythm Society. J Am Coll Cardiol 2014;64:e1-76.

11. Kirchhof P, Benussi S, Kotecha D, et al. 2016 ESC Guidelines for the management of atrial fibrillation developed in collaboration with EACTS. Eur Heart J 2016;37:2893-962.

12. Ogilvie IM, Newton N, Welner SA, et al. Underuse of oral anticoagulants in atrial fibrillation: a systematic review. Am J Med 2010;123:638-45.

13. Public Health England. CVD: Primary Care Intelligence Packs, NHS South Norfolk CCG. 2017. https://wwwgovuk/government/uploads/ system/uploads/attachment_data/file/622956/NHS_East_Riding_of_ Yorkshire_CCG_CVD_intelligence_packpdf (accessed 24 Oct 2017).

14. Gadsbøll K, Staerk L, Fosbøl EL, et al. Increased use of oral anticoagulants in patients with atrial fibrillation: temporal trends from 2005 to 2015 in Denmark. Eur Heart J 2017;38:899-906.

15. Rodríguez-Bernal CL, Hurtado I, García-Sempere A, et al. Oral anticoagulants initiation in patients with atrial fibrillation: real-world data from a population-based cohort. Front Pharmacol 2017;8:63.

16. Freedman B, Camm J, Calkins $\mathrm{H}$, et al. Screening for atrial fibrillation: a report of the AF-SCREEN international collaboration. Circulation 2017;135:1851-67.

17. Mairesse GH, Moran P, Van Gelder IC, et al. Screening for atrial fibrillation: a European Heart Rhythm Association (EHRA) consensus document endorsed by the Heart Rhythm Society (HRS), Asia Pacific Heart Rhythm Society (APHRS), and Sociedad Latinoamericana de Estimulación Cardíaca y Electrofisiología (SOLAECE). Europace 2017;19:1589-623.

18. Tieleman RG, Plantinga Y, Rinkes $D$, et al. Validation and clinical use of a novel diagnostic device for screening of atrial fibrillation. Europace 2014;16:1291-5.
19. Morgan S, Mant D. Randomised trial of two approaches to screening for atrial fibrillation in UK general practice. $\mathrm{Br} J$ Gen Pract 2002;52:373-80.

20. Orchard J, Freedman SB, Lowres N, et al. iPhone ECG screening by practice nurses and receptionists for atrial fibrillation in general practice: the GP-SEARCH qualitative pilot study. Aust Fam Physician 2014;43:315-9.

21. Orchard J, Lowres N, Freedman SB, et al. Screening for atrial fibrillation during influenza vaccinations by primary care nurses using a smartphone electrocardiograph (iECG): A feasibility study. Eur J Prev Cardiol 2016;23:13-20.

22. Britt $\mathrm{H}$, Miller GC, Henderson J, et al. General practice activity in Australia 2015-16: BEACH: bettering the evaluation and care of health. Sydney: Sydney University Press, 2016.

23. Media Release, Prime Minister and Minister for Health. Groundbreaking flu vaccines to protect millions of Aussies. 2018. http:// www.health.gov.au/internet/ministers/publishing.nsf/Content/healthmediarel-yr2018-hunt020.htm (accessed 9 Mar 2018).

24. NSW Government. Shingles vaccination program 2018. http:// wwwhealthnswgovau/immunisation/Pages/Shingles-programaspx (accessed 9 Mar 2018).

25. Australian Government Department of Health. Health assessment for people aged 75 years and older. 2014. http://wwwhealthgovau/ internet/main/publishingnsf/Content/mbsprimarycare_ mbsitem_75andolder (accessed 19 Mar 2018).

26. Australian Government Department of Health. Chronic disease management - provider information. 2016. http://wwwhealthgovau/ internet/main/publishingnsf/content/mbsprimarycare-factsheetchronicdiseasehtm (accessed 9 Mar 2018).

27. Gavino Al, McLachlan CS. Review of screening studies for atrial fibrillation in rural populations of 11 countries. Proc 2017;30:280-5.

28. Australian Institute of Health and Welfare (AlHW). Cardiovascular medicines and primary health care: a regional analysis. Canberra: Australian Institute of Health and Welfare, 2010.

29. Allenby A, Kinsman L, Tham R, et al. The quality of cardiovascular disease prevention in rural primary care. Aust $J$ Rural Health 2016;24:92-8.

30. Peiris D, Usherwood T, Panaretto K, et al. The Treatment of cardiovascular Risk in Primary care using Electronic Decision supOrt (TORPEDO) study-intervention development and protocol for a cluster randomised, controlled trial of an electronic decision support and quality improvement intervention in Australian primary healthcare. BMJ Open 2012;2:e002177.

31. Cadilhac DA, Dewey HM, Vos T, et al. The health loss from ischemic stroke and intracerebral hemorrhage: evidence from the North East Melbourne Stroke Incidence Study (NEMESIS). Health Qual Life Outcomes 2010;8:49.

32. Lowres N, Neubeck L, Redfern J, et al. Community screening programs to identify unknown atrial fibrillation: a systematic review. Eur Heart J 2012;33:61.

33. Lowres N, Neubeck L, Salkeld G, et al. Feasibility and costeffectiveness of stroke prevention through community screening for atrial fibrillation using iPhone ECG in pharmacies. The SEARCH-AF study. Thromb Haemost 2014;111:1167-76.

34. Briggs $\mathrm{AH}$. Statistical approaches to handling uncertainty in health economic evaluation. Eur J Gastroenterol Hepatol 2004;16:551-61.

35. Pawson R, Tilley N. Realistic evaluation. London, UK: Sage Publications, 1997

36. Chan AW, Tetzlaff JM, Altman DG, et al. SPIRIT 2013 statement: defining standard protocol items for clinical trials. Ann Intern Med 2013;158:200-7.

37. Eckman MH, Lip GY, Wise RE, et al. Impact of an atrial fibrillation decision support tool on thromboprophylaxis for atrial fibrillation. Am Heart J 2016;176:17-27.

38. Jackson SL, Peterson GM, Vial JH. A community-based educational intervention to improve antithrombotic drug use in atrial fibrillation. Ann Pharmacother 2004;38:1794-9.

39. Das M, Panter L, Wynn GJ, et al. Primary care atrial fibrillation service: outcomes from consultant-led anticoagulation assessment clinics in the primary care setting in the UK. BMJ Open 2015;5:e009267.

40. McAlister FA, Man-Son-Hing M, Straus SE, et al. Impact of a patient decision aid on care among patients with nonvalvular atrial fibrillation: a cluster randomized trial. CMAJ 2005;173:496-501. 